\title{
CT Angiographic Features of Symptom-Producing Plaque in Moderate-Grade Carotid Artery Stenosis
}

\author{
A. Gupta, E.E. Mtui, H. Baradaran, G. Salama, A. Pandya, H. Kamel, A. Giambrone, and P.C. Sanelli
}

\begin{abstract}
BACKGROUND AND PURPOSE: Emerging evidence indicates that plaque imaging can improve stroke risk stratification in patients with carotid artery atherosclerosis. We studied the association between soft and hard (calcified) plaque thickness measurements on CTA and symptomatic disease status (ipsilateral stroke or TIA) in patients with moderate-grade carotid artery stenosis.
\end{abstract}

MATERIALS AND METHODS: We measured soft-plaque and hard-plaque thickness on CTA axial source images in each carotid artery plaque in subjects with NASCET 50\%-69\% ICA stenosis. We used logistic regression and receiver operating characteristic analyses to assess the strength of the association between thickness measurements and prior stroke or TIA.

RESULTS: Twenty of 72 vessels studied (27.7\%) had ischemic symptoms ipsilateral to the side of moderate-grade carotid stenosis. Each 1 -mm increase in soft plaque resulted in a 3.7 times greater odds of a prior ipsilateral ischemic event $(95 \% \mathrm{Cl}, 1.9-7.2)$. Conversely, for each 1 -mm increase in hard plaque, the odds of being symptomatic decreased by approximately $80 \%(\mathrm{OR}, 0.22 ; 95 \% \mathrm{Cl}, 0.10 \%-0.48 \%)$. Receiver operating characteristic analysis showed an area under the curve of 0.88 by using soft-plaque thickness measurements to discriminate between asymptomatic and symptomatic plaques. Sensitivity and specificity were optimized by using a maximum soft-plaque thickness of $2.2 \mathrm{~mm}$, which provided a sensitivity of $85 \%$ and a specificity of $83 \%$.

CONCLUSIONS: Simple CTA plaque-thickness measurements might differentiate symptomatic and asymptomatic moderate-grade carotid artery plaque. With further prospective validation, CTA plaque measures could function as an easily implementable tool for risk stratification in carotid artery disease.

ABBREVIATIONS: $A U C=$ area under the curve; ROC = receiver operating characteristic

M oderate-grade carotid artery stenosis is a risk factor for stroke, with pooled analysis from randomized controlled trials, including the European Carotid Surgery Trial, NASCET, and Veterans Affairs Symptomatic Trial, suggesting that unoperated patients with moderate-grade stenosis face up to a $20 \%$ 5 -year risk of stroke. ${ }^{1}$ Data pooled from these randomized con-

Received June 18, 2014; accepted after revision July 14.

From the Departments of Radiology (A. Gupta, E.E.M., H.B., G.S., A.P., P.C.S.), Neurology (H.K.), and Healthcare Policy and Research (A.P., A. Giambrone, P.C.S.) and Brain and Mind Research Institute (A. Gupta, H.K.), Weill Cornell Medical College, New York, New York.

Dr Gupta was supported, in part, by the Association of University Radiologists General Electric Radiology Research Academic Fellowship and the 2014-2015 Foundation of the American Society of Neuroradiology Scholar Award.

Paper previously presented in abstract form at: Annual Meeting of the American Society of Neuroradiology and the Foundation of the ASNR Symposium, May 17-22, 2014; Montreal, Quebec, Canada.

Please address correspondence to Ajay Gupta, MD, Department of Radiology, Weill Cornell Medical College 525 East 68th St, Starr 8A, Box 141, New York, NY 10065; e-mail: ajg9004@med.cornell.edu

http://dx.doi.org/10.3174/ajnr.A4098 trolled trials have also shown a modest stroke-risk-reduction benefit from carotid endarterectomy in patients with 50\%-69\% extracranial ICA stenosis. ${ }^{1}$ However, the validity of these data has been questioned in the years since these trials were performed secondary to recent improvements in medical therapy, which have substantially reduced annual stroke rates in patients with carotid disease. ${ }^{2,3}$ Consequently, there has been significant recent effort to develop new imaging markers that can identify patients with carotid artery disease at highest risk for stroke beyond luminal-diameter stenosis measurements.

The 2 main pathophysiologic mechanisms underlying stroke risk in carotid artery disease are the propensity of plaque to locally embolize $^{4,5}$ and downstream hemodynamic compromise (low flow). ${ }^{4,6,7}$ Although hypoperfusion and resultant slow flow from carotid disease likely play a role in a subset of strokes occurring in carotid stenosis, plaque instability causing distal embolus may be a relatively more influential etiologic factor for stroke in carotid disease in general. ${ }^{8}$ Although multisequence MR imaging characterization of plaque has allowed the in vivo discrimination of high-risk plaque tissue elements, ${ }^{9-11}$ its use in clinical practice has 
been limited, given the time, expense, and challenge of performing multisequence carotid plaque MR imaging by using a dedicated carotid coil. CTA-based measurements of soft and hard (calcified) plaque determined on axial CTA source images have recently been proposed as potential simple alternative markers of vulnerable plaque in high-grade ICA stenosis. ${ }^{12,13}$ Because plaque volumes are relatively smaller in moderate-grade stenosis, it is unclear to what extent such techniques are capable of identifying symptom-producing plaque in this group of patients in whom optimal treatment guidelines are controversial. Therefore, we used a cross-sectional study design to assess the association between plaque thickness measurements obtained from neck CTA and previous stroke or TIA in patients with moderate-grade ICA stenosis.

\section{MATERIALS AND METHODS \\ Subjects}

This study was approved by the Human Subjects Institutional Review Board of our institution. We performed a retrospective study with a cross-sectional design by analyzing all CTA neck studies from our institution performed from May 2011 through March 2014. We applied the following inclusion criteria to determine our final cohort: 1) moderate-grade $(50 \%-69 \%)$ extracranial internal carotid artery stenosis as measured on CTA neck examination; 2) adequate documentation in the electronic medical records to determine whether stroke or TIA had occurred before CTA, including review of brain MR imaging or CT; and 3) detailed medical record documentation of pre-existing vascular risk factors. We screened for eligible subjects for study inclusion if the clinical radiology report described a stenosis between $50 \%$ and $69 \%$. We excluded nondiagnostic cases in which a NASCET-style carotid artery stenosis measurement could not be provided during the initial clinical interpretation secondary to significant motion artifacts or suboptimal contrast enhancement of the arterial vasculature.

\section{Imaging Technique}

The CTA neck examinations in this study were performed by using a standardized clinical protocol on one of several scanners at our imaging sites including LightSpeed Pro-16 or HD-750 (GE Healthcare, Milwaukee, Wisconsin). CT was performed in a helical mode with coverage extending from the aortic arch to the $\mathrm{C} 1$ ring. Collimation was performed at $0.625 \mathrm{~mm}$, with a $\mathrm{kV}$ (peak) of 120, auto-milliampere, and a rotation time of 0.5 seconds. Approximately $90 \mathrm{~mL}$ of nonionic iodinated contrast was administered to each patient via an 18-ga peripheral IV catheter at $4-5 \mathrm{~mL} / \mathrm{s}$ by using a power injector and a SmartPrep software (GE Healthcare) region of interest on the aortic arch. In addition, maximum-intensity-projection reconstructions (8-mm thickness with 2-mm intervals) in both sagittal and coronal images were constructed as part of this protocol. To simulate clinical practice imaging conditions, we included, in our analysis, all studies with image quality sufficient to provide clinical interpretation at the time of original image acquisition.

\section{Image Analysis}

The details for CTA neck image analysis were adapted from a previously reported method. ${ }^{12,13}$ Briefly, a board-certified neuroradiologist analyzed axial source images from the CTA examination and recorded 3 measurements for each carotid artery: 1)

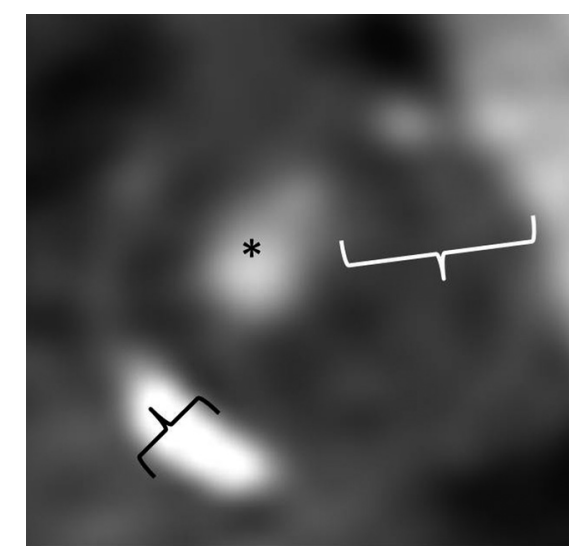

FIG 1. Representative magnified axial CTA image of the proximal internal carotid artery in a patient with predominantly soft plaque. The asterisk represents the residual patent ICA lumen. The white calipers represent maximum linear soft-plaque thickness, and black calipers represent maximum hard-plaque thickness measurements.

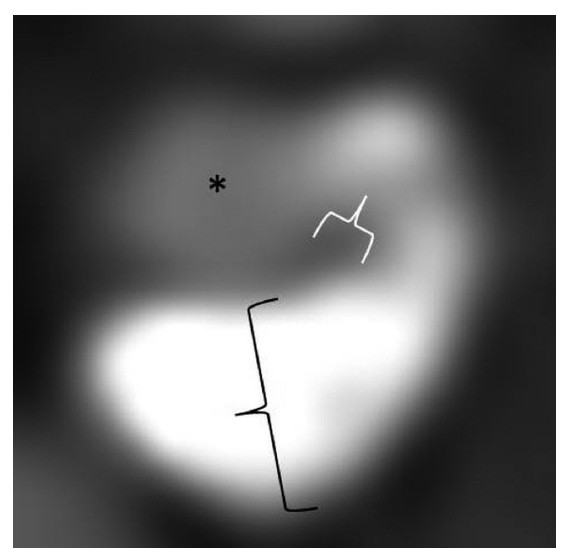

FIG 2. Representative magnified axial CTA image of the proximal internal carotid artery in a patient with predominantly calcified plaque. The asterisk represents the residual patent ICA lumen. The white calipers represent maximum linear soft-plaque thickness, and black calipers represent maximum hard-plaque thickness measurements.

NASCET stenosis measurements by using the normal distal ICA as the denominator for stenosis calculation; 2) maximum thickness of the noncalcified, soft-plaque component on the single axial section on which luminal diameter stenosis was greatest; and 3) maximum thickness of the calcified, hard-plaque component on the same single axial section on which luminal-diameter stenosis was greatest (see representative measurements in Figs 1 and 2 ). Plaques that were partially calcified and partially soft in attenuation had both maximum thickness measurements taken on the same single-axial section with the greatest luminal stenosis. These linear measurements were obtained by using the electronic caliper function of Centricity PACS (GE Healthcare), which allows measurements with a spatial resolution to $0.1 \mathrm{~mm}$. Although the reported NASCET percentage stenosis in the existing clinical radiology report was used to screen for eligibility, all NASCET measurements used in the analysis of the included cohort were remeasured by a study investigator blinded to all clinical data to ensure strict compliance with NASCET methodology. ${ }^{14,15}$ These repeat study measurements were performed with care to avoid distal luminal denominator measurements in near-occlusion, 
Table 1: Cohort demographics and risk-factor differences between main study groups ${ }^{a}$

\begin{tabular}{lccc}
\hline & $\begin{array}{c}\text { Asymptomatic } \\
(\boldsymbol{n}=\mathbf{5 2})\end{array}$ & $\begin{array}{c}\text { Symptomatic } \\
(\boldsymbol{n}=\mathbf{2 0})\end{array}$ & $\boldsymbol{P}$ Value \\
\hline $\begin{array}{l}\text { Demographic } \\
\text { Age (yr) }\end{array}$ & $74.1 \pm 9.8$ & $74.2 \pm 11.6$ & .9786 \\
$\quad$ Male & $52 \%(27)$ & $70 \%(14)$ & .1653 \\
CT & $59.0 \pm 3.4 \%$ & $56.6 \pm 6.1 \%$ & .1780 \\
$\quad$ Stenosis & & & \\
Cardiovascular risk factors & $62 \%(32)$ & $45 \%(9)$ & .2043 \\
$\quad$ Smoking history & $90 \%(47)$ & $80 \%(16)$ & .2327 \\
$\quad$ Hypertension & $73 \%(38)$ & $65 \%(13)$ & .4994 \\
Hyperlipidemia & $25 \%(13)$ & $20 \%(4)$ & .7640 \\
$\quad$ Diabetes & $54 \%(28)$ & $35 \%(7)$ & .1518 \\
$\quad$ Coronary artery disease & $23 \%(12)$ & $40 \%(8)$ & .1518 \\
$\quad$ Atrial fibrillation & & & \\
CTA imaging markers & $2.69 \pm 1.04$ & $1.19 \pm 0.92$ & $<.0001$ \\
$\quad$ Max hard-plaque thickness (mm) & $1.41 \pm 0.97$ & $3.45 \pm 1.54$ & $<.0001$ \\
Max soft-plaque thickness (mm) & $0.62 \pm .57$ & $2.73 \pm 1.95$ & $<.0001$ \\
$\quad$ Ratio soft-/hard-plaque thickness & & & \\
\hline Note: Max indicates maximum. & & &
\end{tabular}

Note:-Max indicates maximum.
${ }^{a}$ Mean values are shown with number of subjects shown in parentheses and SDs shown after \pm signs.

stenosis percentage, sex, age, and any covariate risk factors found to be statistically significant $(P<.05)$ in univariate analyses. The ratio of soft-to-hard plaque was also analyzed. Receiver operating characteristic (ROC) analyses were also performed for each CTA imaging feature (hard plaque, soft plaque, and ratio measurements), and optimum cutoffs were calculated for the plaque measurement shown to have the highest area under the curve (AUC) for the detection of symptomatic plaque. We also calculated operating characteristics of each plaque thickness measurement, including sensitivity, specificity, positive predictive value, and negative predictive value. An optimal cutoff for the plaque measurement shown to have the highest which could incorrectly underestimate stenosis severity. In addition, in cases without near-occlusion, all distal luminal denominator measurements were obtained distal to the tapering carotid bulb in the segment of the extracranial ICA distal to parallel walls.

All measurements were obtained on source axial sections without additional postprocessing. We used these standard axial images instead of orthogonal reconstructions so that we could simulate routinely used clinical imaging protocols and also because excellent agreement between axial and orthogonal plaque element thickness measurements were previously found by using this technique. ${ }^{12}$ We performed our analysis with window/level settings at approximately 800/200 with small adjustments made manually to optimize discrimination between soft and hard plaque. A second neuroradiologist with prior experience using this technique repeated thickness measurements on a subset of 20 subjects to assess measurement reproducibility.

\section{Clinical Data Assessment}

The history of ipsilateral TIA or stroke and pre-existing vascular risk factors were determined by a consensus of 2 study investigators after a detailed examination of the electronic medical record, with any disagreements in assessment resolved by consensus. All clinical data were determined blinded to CTA imaging analysis. We used American Heart Association definitions of TIA and stroke, ${ }^{16}$ with stroke and TIA defined as a permanent or transient episode, respectively, of neurologic dysfunction caused by focal brain or retinal ischemia. We classified stroke or TIA as being symptomatic disease only when events were referable to the stenotic ICA. We also recorded the days between qualifying ipsilateral ischemic events and CTA and the presence of a smoking history, diabetes (a hemoglobin A1C of $>6.5 \%$ or on diabetic medication), hypertension (blood pressure of $>140 / 90 \mathrm{~mm} \mathrm{Hg}$ or on antihypertensive medication), atrial fibrillation, hyperlipidemia (LDL of $>100$ or on statins), and coronary artery disease.

\section{Statistical Analysis}

Multivariate logistic regression analysis was used to examine the association between each 1-mm increase in plaque-thickness measure and symptomatic disease status while adjusting for exact
AUC was also calculated. All statistical analyses were performed by using SAS 9.3 (SAS Institute, Cary, North Carolina).

\section{RESULTS}

\section{Subject Characteristics}

We screened 1376 patients and arrived at our final cohort of 68 subjects after excluding subjects who did not meet inclusion criteria. Four subjects with bilateral moderate-grade stenosis were included, resulting in 72 vessels eligible for analysis. Clinical indications for CTA included suspicion of an acute ischemic event in 32 subjects $(47.1 \%)$, routine imaging for known carotid stenosis in 20 subjects (29.4\%), work-up of abnormal findings on carotid sonography in 7 subjects $(10.3 \%)$, altered mental status in 3 subjects $(4.4 \%)$, neck pain in 2 subjects $(2.9 \%)$, follow-up for a history of contralateral carotid endarterectomy in 2 subjects $(2.9 \%)$, and follow-up for intracranial aneurysm in 2 subjects $(2.9 \%)$.

Twenty of 72 vessels studied (27.7\%) had ischemic symptoms ipsilateral to the side of moderate-grade carotid stenosis. Among the cerebrovascular events that had occurred, 19/20 occurred within 1 month of the CTA examination. Of the total events, 16 were ipsilateral strokes that occurred a median of 0 days before CTA (range, 0-621 days), while 4 were ipsilateral TIAs that occurred a median of 2.5 days before CTA (range, 0-16 days). All 20 patients with ischemic symptoms ipsilateral to the side of moderate-grade stenosis had imaging confirmation with either CT or MR which showed an infarct in all 16 patients with stroke and no evidence of infarct in the 4 subjects with TIA. Patient cohort characteristics are shown in Table 1. Vascular risk factors were not significantly different between groups.

\section{CTA Plaque Imaging Results}

Mean soft-plaque thickness was significantly higher in subjects with symptomatic disease, while mean hard-plaque thickness was significantly higher in asymptomatic subjects (Table 1). We found that each 1-mm increase in mean soft-plaque thickness was associated with a $\sim 3.7$ times greater likelihood of prior stroke or TIA $(P=.0001)$, after adjusting for age, exact percentage stenosis, and 
Table 2: Univariate and multivariate logistic regression odds ratios associating plaque characteristics with symptomatic carotid disease

\begin{tabular}{|c|c|c|c|c|c|c|c|c|c|}
\hline \multirow[b]{2}{*}{ Predictors of Interest } & \multicolumn{4}{|c|}{$\begin{array}{l}\text { Univariate Logistic } \\
\text { Regression }\end{array}$} & \multicolumn{4}{|c|}{$\begin{array}{l}\text { Adjusted Logistic } \\
\text { Regression }^{\mathrm{a}}\end{array}$} & \multirow{2}{*}{$\begin{array}{c}\begin{array}{c}\text { Difference in Univariate } \\
\text { and Adjusted AUC }\end{array} \\
P \text { Value }\end{array}$} \\
\hline & OR & $95 \% \mathrm{Cl}$ & AUC & $P$ Value & OR & $95 \% \mathrm{Cl}$ & AUC & $P$ Value & \\
\hline Max hard-plaque thickness (mm) & 0.22 & $0.10-0.47$ & 0.85 & .0001 & 0.22 & $0.10-0.48$ & 0.87 & .0001 & .5205 \\
\hline Max soft-plaque thickness (mm) & 3.98 & $2.02-7.81$ & 0.87 & $<.0001$ & 3.73 & $1.92-7.24$ & 0.88 & $<.0001$ & .6193 \\
\hline Ratio soft-/hard-plaque thickness & 6.40 & $2.44-16.80$ & 0.87 & .0002 & 6.12 & $2.34-16.02$ & 0.86 & .0002 & .7146 \\
\hline
\end{tabular}

Note:-Max indicates maximum.

${ }^{a}$ Adjusted for age, stenosis (NASCET), and sex.
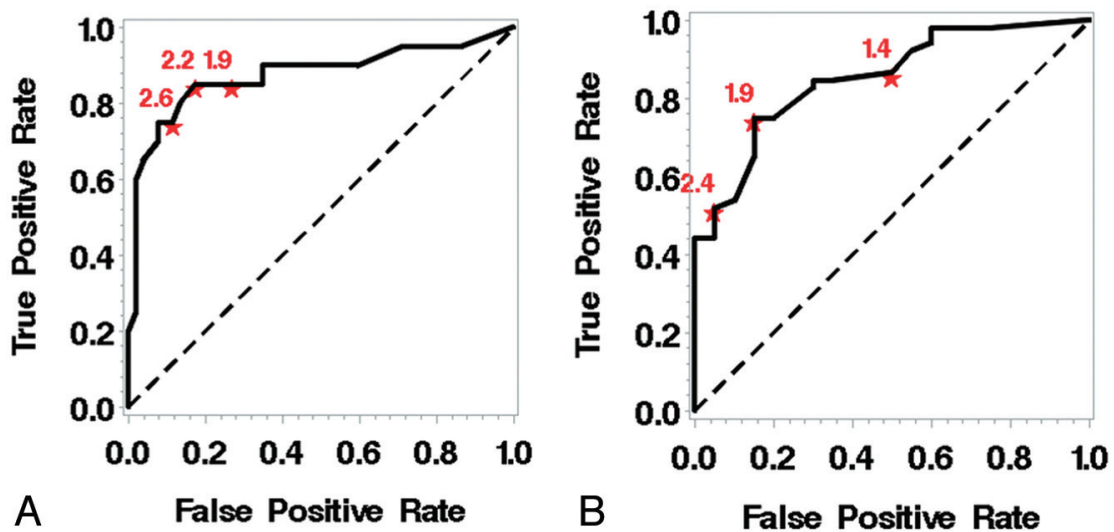

FIG 3. Receiver operating characteristic curves for maximum soft-plaque thickness $(A)$ and maximum hard-plaque thickness $(B)$.

sex (Table 2). Each 1-mm increase in hard-plaque thickness decreased the odds of having had a prior ipsilateral stroke or TIA by approximately $80 \%(\mathrm{OR}=0.22, P=.0001)$. The ratio between soft- and hard-plaque thickness was similarly predictive of symptomatic disease. Given the single outlier ischemic event occurring $>30$ days before CTA imaging (a stroke in 1 subject 621 days before CTA), in a post hoc sensitivity analysis excluding this subject, hardand soft-plaque thickness adjusted-ORs were not significantly changed at 0.22 and 3.77, respectively. We calculated interreader correlation coefficients of 0.97 and 0.96 for soft- and hard-plaque thickness measurements, respectively, consistent with prior reports. ${ }^{12,13}$

\section{Diagnostic Accuracy Measures}

In our ROC analysis adjusted for age, sex, and stenosis severity, soft-plaque thickness measures provided the greatest AUC (0.88) (Table 2 and Fig 3). Sensitivity and specificity were optimized by using a maximum soft-plaque thickness of $2.2 \mathrm{~mm}$, which provided a sensitivity of $85 \%$, specificity of $83 \%$, positive predictive value of $65 \%$, and negative predictive value of $93 \%$. Hard-plaque thickness measurements provided a similar AUC of 0.87. Sensitivity and specificity were optimized by using a maximum hardplaque thickness measurement of $1.9 \mathrm{~mm}$, which provided a sensitivity of $75 \%$, specificity of $85 \%$, positive predictive value of $93 \%$, and negative predictive value of $57 \%$.

\section{DISCUSSION}

Using a simple and reproducible plaque measurement on CTA, we found a strong association between increasing soft-plaque thickness measurements and symptomatic carotid artery plaques. Previous studies have shown that CTA plaque thickness measures can predict high-risk plaque as defined on correlative MR imaging ${ }^{12}$ or symptomatic plaque in high-grade stenosis. ${ }^{13}$ In the current study, we found that such CTA plaque measures can differ- entiate asymptomatic and symptomatic carotid plaques despite the relatively smaller volume of plaque and lower absolute stroke risk present in moderategrade stenosis. Moreover, we found no significant differences in traditional vascular risk factors in the asymptomatic and symptomatic groups, suggesting that plaque thickness measurements may be able to discriminate high-risk and stable plaque more accurately than clinical factors. This is the first report, to our knowledge, applying this CTA technique to patients with a tightly defined and clinically relevant NASCET stenosis category of $50 \%-69 \%$. Although the absolute annual risk of stroke is lower in moderate-grade compared with high-grade carotid artery stenosis, the prevalence of 50\%$69 \%$ stenosis is approximately 3 times higher in the general population ${ }^{17}$; therefore, the total population-based stroke risk attributable to moderate stenosis is substantial.

Although there are studies using alternative CTA imaging strategies to assess unstable or vulnerable carotid plaque, we believe that the technique we used has 3 significant advantages over previously studied CTA techniques. First, plaque thickness measurements require no additional postprocessing, unlike some prior methods ${ }^{18,19}$ that have required customized postprocessing software not routinely available or widely used in clinical practice. Similarly, the technique used in our study does not require the measurement of Hounsfield units, which have been shown to be poor discriminators among specific plaque elements, given overlapping attenuation of in vivo atherosclerotic plaque components such as hemorrhage, fibrosis, and lipids. ${ }^{20,21}$ Second, plaque thickness measurements do not require additional imaging acquisitions to be obtained as part of the CTA examination. Romero et $\mathrm{al}^{22}$ for example, showed a correlation between arterial wall enhancement and symptomatic plaque, but their approach necessitated a noncontrast CT of the neck, an acquisition that is not part of most CTA neck studies. Third, plaque thickness measurements are highly reproducible, as evidenced by the excellent interobserver correlation coefficients we and others have found. ${ }^{12} \mathrm{Al}$ though assessment of plaque morphology such as ulceration on CTA has been proposed as a high-risk plaque marker, ${ }^{20,23}$ the technique used in our study may be more desirable to implement in routine clinical image interpretation because it is less prone to observer subjectivity.

The mechanism underlying increased risk of symptomatic disease in carotid atheroma with greater soft plaque is not entirely 
understood but is likely related to the low attenuation of high-risk elements of atherosclerosis, including a lipid-rich necrotic core and intraplaque hemorrhage. ${ }^{24}$ The precise differentiation of tissue components within soft plaque may be of limited clinical significance, however, because most of these presumed tissue types are features of more advanced atherosclerotic lesions. ${ }^{11}$ Understanding the relative importance of specific carotid plaque elements in predicting stroke risk has been made possible by highresolution MR imaging. By allowing the accurate differentiation of tissue types in atherosclerotic lesions, ${ }^{12} \mathrm{MR}$ imaging provides important insights into the magnitude of risk that could potentially be conferred by increasing soft plaque on CTA. For example, a recent meta-analysis ${ }^{25}$ of subjects with carotid stenosis followed after plaque MR imaging showed a lipid-rich necrotic core, intraplaque hemorrhage, and thinning or rupture of the fibrous cap as conferring 3-, 6-, and 4.5-fold higher risks of future stroke or TIA, respectively. Because lipid cores or hemorrhage together likely form a significant component of soft plaque, ${ }^{13}$ we hypothesize that soft-plaque measurements may represent a simple composite marker of vulnerable, high-risk plaque elements. Similarly, calcium in carotid plaque may be associated with relatively more stable plaque, which is likely to cause ischemic stroke. The apparent protective effect afforded by increasing calcium burden that we and other investigations ${ }^{12,13,26}$ have found might be explained by the decrease in fibrous cap inflammation or the increase in mechanical stability afforded by attenuated calcium deposition. ${ }^{26}$

Limitations of this study require discussion. First, because it was a cross-sectional retrospective analysis, prior ischemic events were correlated with plaque imaging features rather than prediction of future stroke or TIA. Second, we were not able to precisely identify the tissue types constituting soft plaque on the basis of our study, which lacked histopathologic validation. Third, although the review of subject data makes carotid disease the most likely cause of stroke or TIA in our cohort, the possibility of tandem intracranial stenosis as a cause of ischemic symptoms in our cohort cannot be entirely excluded because we studied the neck arteries and not the intracranial circulation. We believe that our current study and prior work ${ }^{12,13}$ now justify a larger scale, appropriately powered prospective investigation of the ability of CTA plaque-thickness measurements to predict future stroke or TIA. Because plaque hemorrhage and lipid-rich necrotic core are powerful predictors of stroke on MR imaging, ${ }^{25}$ it is likely that similar predictive information would be present in CTA attenuation measurements because we believe that hemorrhage and lipid are significant constituents of soft plaque. Moreover, although carotid atherosclerosis is a dynamic process, prospective evidence supports high-risk markers of plaque, such as intraplaque hemorrhage, as being relatively stable with time and conferring increased stroke risk for at least 5 years after their detection. ${ }^{5}$ An additional recent study ${ }^{27}$ also showed that high-risk elements such as intraplaque hemorrhage, fibrous cap abnormalities, and lipid cores were generally not significantly changed during a 1 -year follow-up period. Therefore, although further investigation is needed, it is reasonable to hypothesize that our results could translate into a prospective CTA-based stroke prediction tool in carotid artery disease.

\section{CONCLUSIONS}

By using simple single-axial source images from CTA, we were able to use soft- and hard-thickness measurements to discriminate between asymptomatic and symptomatic carotid artery plaque in a cohort of patients with moderate carotid artery stenosis. Ultimately, further prospective studies powered for stroke prediction are needed using this relatively simple and reproducible CT-based method.

Disclosures: Ajay Gupta—RELATED: Grant: Association of University Radiologists-GE Radiology Research Academic Fellowship Award, ${ }^{*}$ Foundation of the American Society of Neuroradiology Scholar Award. * Money paid to the institution.

\section{REFERENCES}

1. Rothwell PM, Eliasziw M, Gutnikov SA, et al. Analysis of pooled data from the randomised controlled trials of endarterectomy for symptomatic carotid stenosis. Lancet 2003;361:107-16

2. Abbott AL. Medical (nonsurgical) intervention alone is now best for prevention of stroke associated with asymptomatic severe carotid stenosis: results of a systematic review and analysis. Stroke 2009; $40:$ e573-83

3. Raman G, Moorthy D, Hadar N, et al. Management strategies for asymptomatic carotid stenosis: a systematic review and meta-analysis. Ann Intern Med 2013;158:676-85

4. Caplan LR, Hennerici M. Impaired clearance of emboli (washout) is an important link between hypoperfusion, embolism, and ischemic stroke. Arch Neurol 1998;55:1475-82

5. Hosseini AA, Kandiyil N, Macsweeney ST, et al. Carotid plaque hemorrhage on magnetic resonance imaging strongly predicts recurrent ischemia and stroke. Ann Neurol 2013;73:774-84

6. Derdeyn CP. Mechanisms of ischemic stroke secondary to large artery atherosclerotic disease. Neuroimaging Clin N Am 2007;17:30311, vii-viii.

7. Gupta A, Chazen JL, Hartman M, et al. Cerebrovascular reserve and stroke risk in patients with carotid stenosis or occlusion: a systematic review and meta-analysis. Stroke 2012;43:2884-91

8. Morgenstern LB, Fox AJ, Sharpe BL, et al. The risks and benefits of carotid endarterectomy in patients with near occlusion of the carotid artery: North American Symptomatic Carotid Endarterectomy Trial (NASCET) group. Neurology 1997;48:911-15

9. den Hartog AG, Bovens SM, Koning W, et al. Current status of clinical magnetic resonance imaging for plaque characterisation in patients with carotid artery stenosis. Eur J Vasc Endovasc Surg 2013;45:7-21

10. Cai JM, Hatsukami TS, Ferguson MS, et al. Classification of human carotid atherosclerotic lesions with in vivo multicontrast magnetic resonance imaging. Circulation 2002;106:1368-73

11. Stary HC, Chandler AB, Dinsmore RE, et al. A definition of advanced types of atherosclerotic lesions and a histological classification of atherosclerosis: a report from the committee on vascular lesions of the council on arteriosclerosis, American Heart Association. Circulation 1995;92:1355-74

12. Trelles M, Eberhardt KM, Buchholz M, et al. CTA for screening of complicated atherosclerotic carotid plaque-American Heart Association type VI lesions as defined by MRI. AJNR Am J Neuroradiol 2013;34:2331-37

13. Gupta A, Baradaran H, Kamel H, et al. Evaluation of computed tomography angiography plaque thickness measurements in highgrade carotid artery stenosis. Stroke 2014;45:740-45

14. Fox AJ, Symons SP, Aviv RI, et al. Falsely claiming use of NASCET percentage stenosis method. Radiology 2009;253:574-45, author reply 575

15. Fox AJ. How to measure carotid stenosis. Radiology 1993;186: 316-18

16. Easton JD, Saver JL, Albers GW, et al. Definition and evaluation of transient ischemic attack: a scientific statement for healthcare pro-

AJNR Am J Neuroradiol 36:349-54 Feb 2015 www.ajnr.org

353 
fessionals from the American Heart Association/American Stroke Association Stroke Council; Council on Cardiovascular Surgery and Anesthesia; Council on Cardiovascular Radiology and Intervention; Council on Cardiovascular Nursing; and the Interdisciplinary Council on Peripheral Vascular Disease-the American Academy of Neurology affirms the value of this statement as an educational tool for neurologists. Stroke 2009;40:2276-93

17. de Weerd M, Greving JP, Hedblad B, et al. Prevalence of asymptomatic carotid artery stenosis in the general population: an individual participant data meta-analysis. Stroke 2010;41:1294-97

18. Wintermark M, Jawadi SS, Rapp JH, et al. High-resolution CT imaging of carotid artery atherosclerotic plaques. AJNR Am J Neuroradiol 2008;29:875-82

19. Wintermark M, Arora S, Tong E, et al. Carotid plaque computed tomography imaging in stroke and nonstroke patients. Ann Neurol 2008;64:149-57

20. U-King-Im JM, Fox AJ, Aviv RI, et al. Characterization of carotid plaque hemorrhage: a CT angiography and MR intraplaque hemorrhage study. Stroke 2010;41:1623-29

21. Walker LJ, Ismail A, McMeekin W, et al. Computed tomography angiography for the evaluation of carotid atherosclerotic plaque: correlation with histopathology of endarterectomy specimens. Stroke 2002;33:977-81

22. Romero JM, Babiarz LS, Forero NP, et al. Arterial wall enhancement overlying carotid plaque on CT angiography correlates with symptoms in patients with high grade stenosis. Stroke 2009;40:1894-96

23. Hokari M, Kuroda S, Yasuda $\mathrm{H}$, et al. Lumen morphology in mildto-moderate internal carotid artery stenosis correlates with neurological symptoms. J Neuroimaging 2011;21:348-54

24. El-Barghouty NM, Levine T, Ladva S, et al. Histological verification of computerised carotid plaque characterisation. Eur J Vasc Endovasc Surg 1996;11:414-16

25. Gupta A, Baradaran H, Schweitzer AD, et al. Carotid plaque MRI and stroke risk: a systematic review and meta-analysis. Stroke 2013; 44:3071-77

26. Kwee RM. Systematic review on the association between calcification in carotid plaques and clinical ischemic symptoms. J Vasc Surg 2010;51:1015-25

27. Kwee RM, Truijman MT, van Oostenbrugge RJ, et al. Longitudinal MRI study on the natural history of carotid artery plaques in symptomatic patients. PLoS One 2012;7:e42472 